\title{
Poikiloderma atrophicans vasculare as a pointer to reticulosis of the skin
}

\author{
ROBERT S. ChapmaN \\ M.B., M.R.C.P. \\ C. Jose Paul \\ M.B., B.S.
}

Department of Dermatology, Stobhill General Hospital, Glasgow G21 3UW

\begin{abstract}
Summary
A case of reticulosis of the skin (mycosis fungoides) terminating in reticulum cell sarcoma is described where the features of poikiloderma atrophicans vasculare had been present for $\mathbf{4 0}$ years. The likelihood of reticulosis eventually developing after such a change in the skin is believed to be around $50 \%$.
\end{abstract}

AN important location of reticulo-endothelial tissue is the dermis of the skin (Robb-Smith, 1944). As in other sites, this tissue can undergo proliferation, ultimately to give a lymphoma, the clinical presentation being termed mycosis fungoides by dermatologists. Evolution is slow, 20 years or more usually elapsing before frank malignancy occurs. At this stage the fungating skin tumours which Alibert first described in the early 19th century may appear.

Poikiloderma atrophicans vasculare of the skin, defined as pigmentation, telangiectasia, light scaling and epidermal atrophy giving so-called cigarettepaper skin, was reported by Lane in 1923 to precede Hodgkin's disease. Another case mentioned by him in 1921 , also with poikiloderma, died eventually from lymphosarcoma. Increasing evidence would suggest that while poikiloderma may be found in one or two inherited disorders, in dermatomyositis and as a consequence of radiotherapy or of actinic damage, its main importance is as an indicator of present or future disorder of the reticulo-endothelial system of the skin. Samman (1964) found that of twenty-five cases with poikiloderma, twelve were showing progression towards a reticulosis. In 1968, he reported that fourteen of the twenty-five were showing such progress while of thirty-five additional cases eighteen were following a similar course towards reticulosis.

We wish to report a case where poikiloderma atrophicans vasculare was present for 40 years, to be followed by mycosis fungoides and terminal reticulum cell sarcoma.

\section{Case report}

A man of 63 presented in 1972 with a history of progressively extending areas of redness and scaling on his trunk from his early twenties. In 1970, ulcers appeared on his skin, mainly at sites of friction.
Initially, these ulcers healed with simple dressings. Later ulcers were more stubborn, leading him to seek advice.

\section{Examination}

This showed striking pigmentation of skin on trunk, face and arms, scars at the sites of previous ulceration (Fig. 1) and infiltration of deeper layers of the skin. Closer examination (Fig. 2) showed the features of poikiloderma atrophicans vasculare including the thin epidermis which could easily be picked up between finger and thumb. A large ulcer over the right scapula (Fig. 3) showed a yellow indolent base, very slightly pouting edges and firmly adherent black eschar. Examination was otherwise unremarkable, there being no hepatosplenomegaly and no lymphadenopathy of note except for small, discrete nodes in the axillae. These were of the size often found in patients with extensive skin disease and resulting in what is termed dermopathic lymphadenopathy. Pruritus was not experienced at this time nor during the rest of his illness. Clinically, we considered the patient to show poikiloderma atrophicans vasculare with underlying skin reticulosis.

\section{Investigations}

ESR $36 \mathrm{~mm} / \mathrm{hr}, \mathrm{Hb} 14.6 \mathrm{~g} / 100 \mathrm{ml}$, WBC $7500 / \mathrm{mm}^{3}$ with $81 \%$ neutrophils, $2 \%$ eosinophils, $14 \%$ lymphocytes and 3\% monocytes. Sternal marrow showed no abnormality, erythropoiesis being normoblastic. Plasma cells and reticulum cells were within normal limits. Urea, electrolytes, serum proteins, strip electrophoresis and liver function tests were normal. Serum IgA was slightly raised at $323 \mathrm{mg} \%$ but IgG and IgM were within normal limits. Coliforms, staphylococci and streptococci were repeatedly grown on culture from skin ulcers.

Histological examination of skin biopsies showed atrophy of epidermis with liquefaction degeneration of the basal layer. There was marked sub-epidermal oedema and marked telangiectasia of dermal blood vessels. In the upper dermis there was a dense inflammatory infiltrate composed of histiocytes, lymphocytes and a few granulocytes. There were also many pigment-laden macrophages. Mycosis fungoides or lymphoma was strongly suggested. 


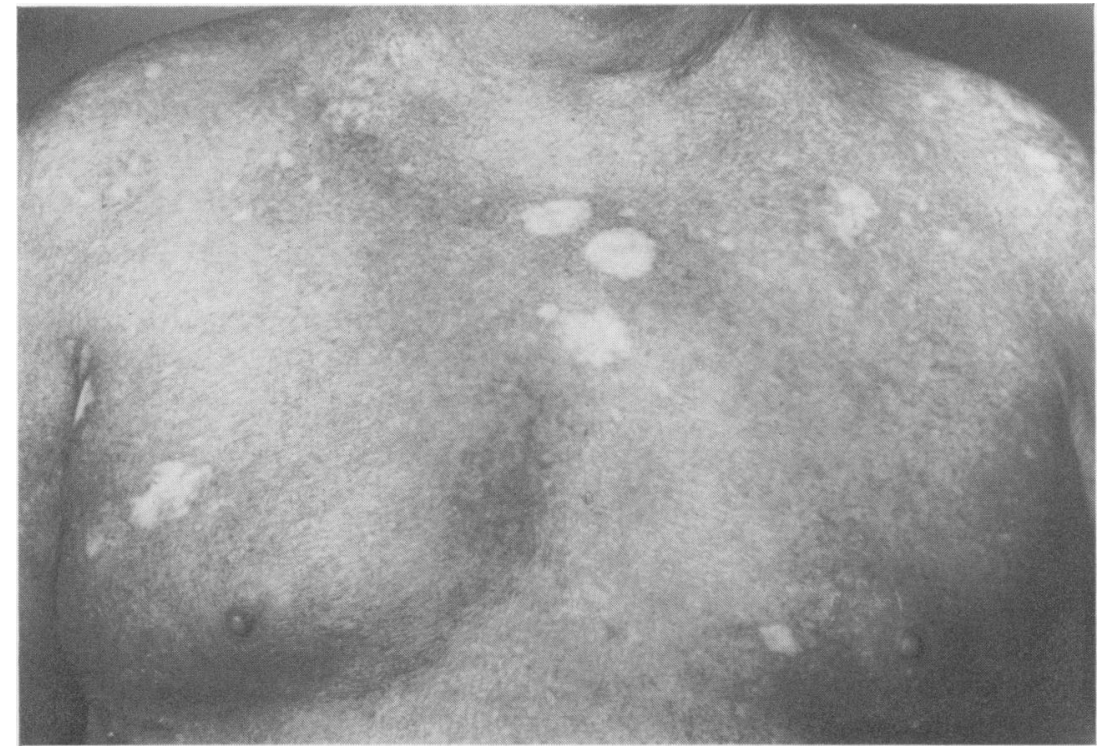

FIG. 1. Dusky pigmentation over trunk with scars at sites of previous ulceration.

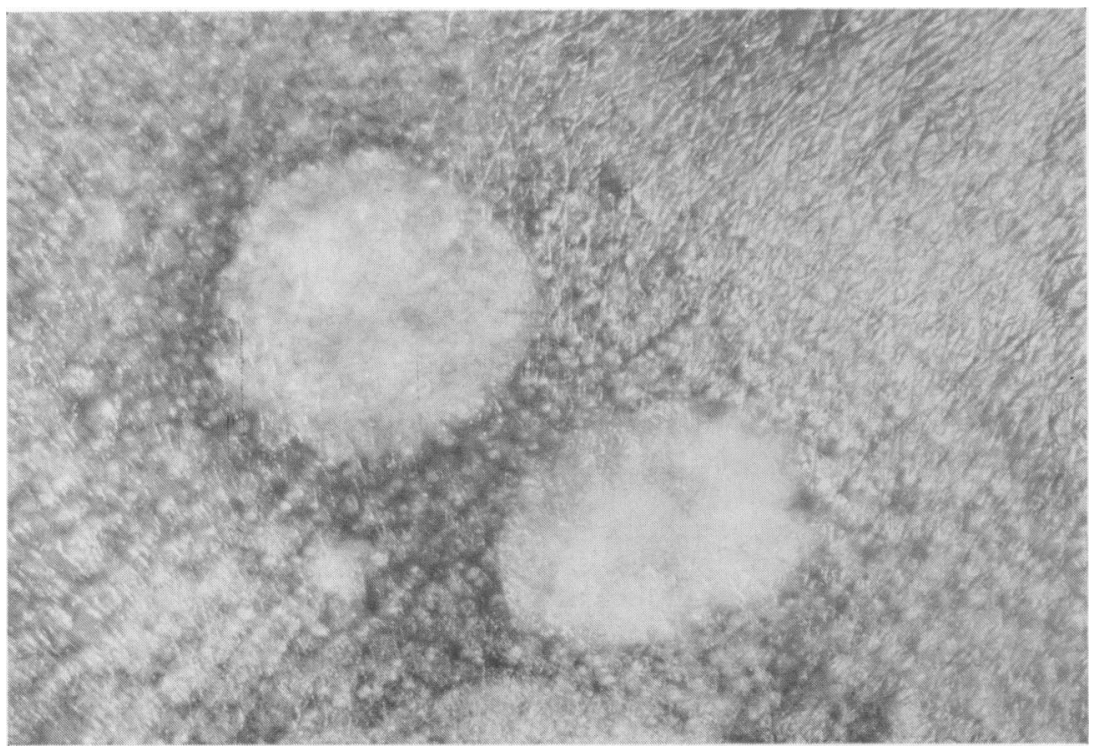

FIG. 2. Detail of skin showing pigmentation, loose wrinkled surface and healed ulcers. 


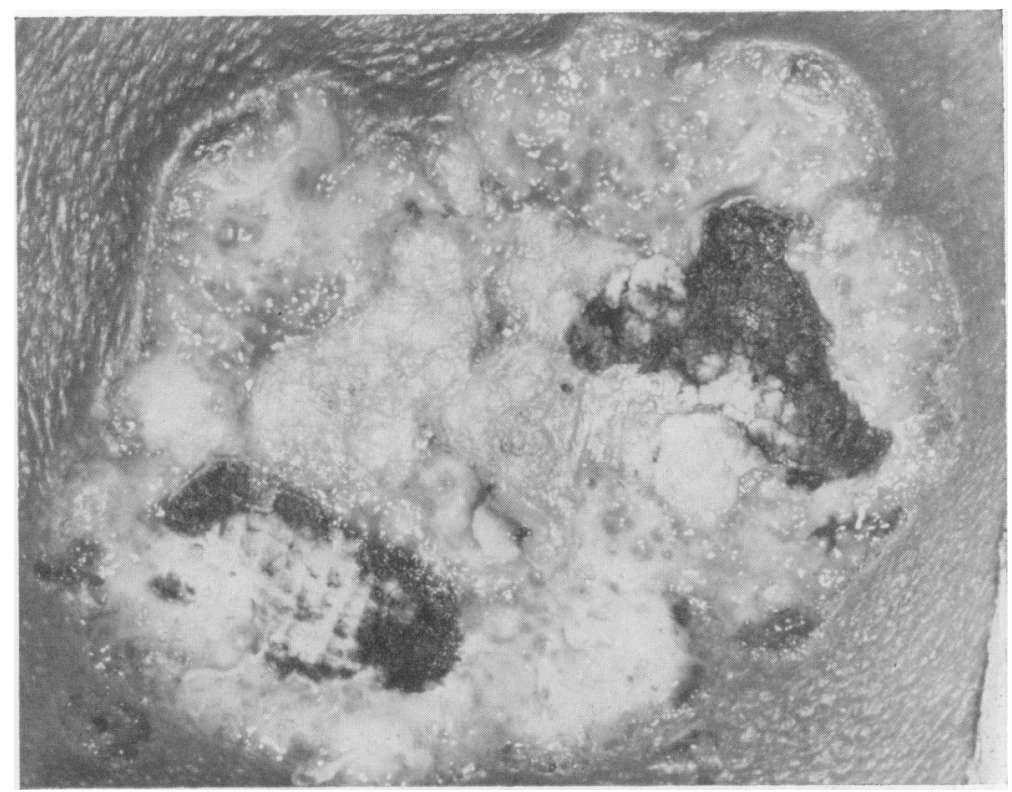

FIG. 3. Indolent ulcer with black eschar and slightly pouting margin.

\section{Progress}

In view of the lengthy course of the patient's illness, radiotherapy and electron beam therapy were considered but rejected temporarily. Conservative management failed to give healing of the ulcers, however, and prednisone in a dose of $20 \mathrm{mg}$ daily was begun. Healing was rapid at all sites. Steroid therapy was continued from then on, dosage being increased to a maximum of $60 \mathrm{mg}$ prednisone daily during episodes of ulceration. Hospital admission was necessary at 4-6 month intervals to ensure adequate care of ulcerated areas. There was never more than a minimal degree of pouting of the edges of such ulcers and no frank tumour formation.

Skin biopsies taken at intervals during the 18month period of the patient's attendance showed features similar to those in the initial biopsy, the dermal infiltrate being composed mainly of lymphoid cells with smaller numbers of eosinophils and histiocytes or reticulum cells, some of which were atypical. A biopsy taken after the patient's final admission in October 1973 showed a considerable increase of the infiltrate with many histiocytes and lymphocytes often with pyknotic nuclei (Fig. 4). Extension of the infiltrate to deeper layers of the dermis was also prominent.

Since there was now little evidence of the steroid being of benefit, prednisone was reduced and finally discontinued early in November 1973. In spite of this, all ulcers were healing satisfactorily apart from one on the upper chest which responded well to superficial radiotherapy. The patient was now experiencing pain in the right hip. Orthopaedic examination indicated disease of the right sacro-iliac joint. X-ray showed erosion of the joint margins but no other abnormality of the pelvis. An isotope scan indicated a considerable increase in uptake by the entire right ilium. Histology of a bone biopsy from the ilium showed the marrow to be replaced by histiocyte-like cells with pink or clear cytoplasm with many fine reticulin fibres between the cells, giving a diagnosis of diffuse histiocytic malignant lymphoma or reticulum cell sarcoma. Sternal marrow was still entirely normal.

Death occurred suddenly 3 weeks later from pulmonary embolism. Autopsy revealed a large mass in the pelvis arising from the right iliac fossa with destruction of bone and invasion of the psoas muscle. Histological examination showed the psoas muscle (Fig. 5) to be partly replaced and partly infiltrated by histiocyte-like cells of uniform size and shape arranged in continuous sheets. The spleen contained nodules composed of cells of varying size and shape separated by reticulin fibres. Thyroid gland and abdominal lymph nodes showed similar features. Axillary and inguinal nodes showed no evidence of neoplasm. The liver showed some fatty change. No other organs were involved. The final pathological diagnosis was pleomorphic reticulum cell sarcoma or malignant diffuse nodular pleomorphic histiocytic lymphoma. 


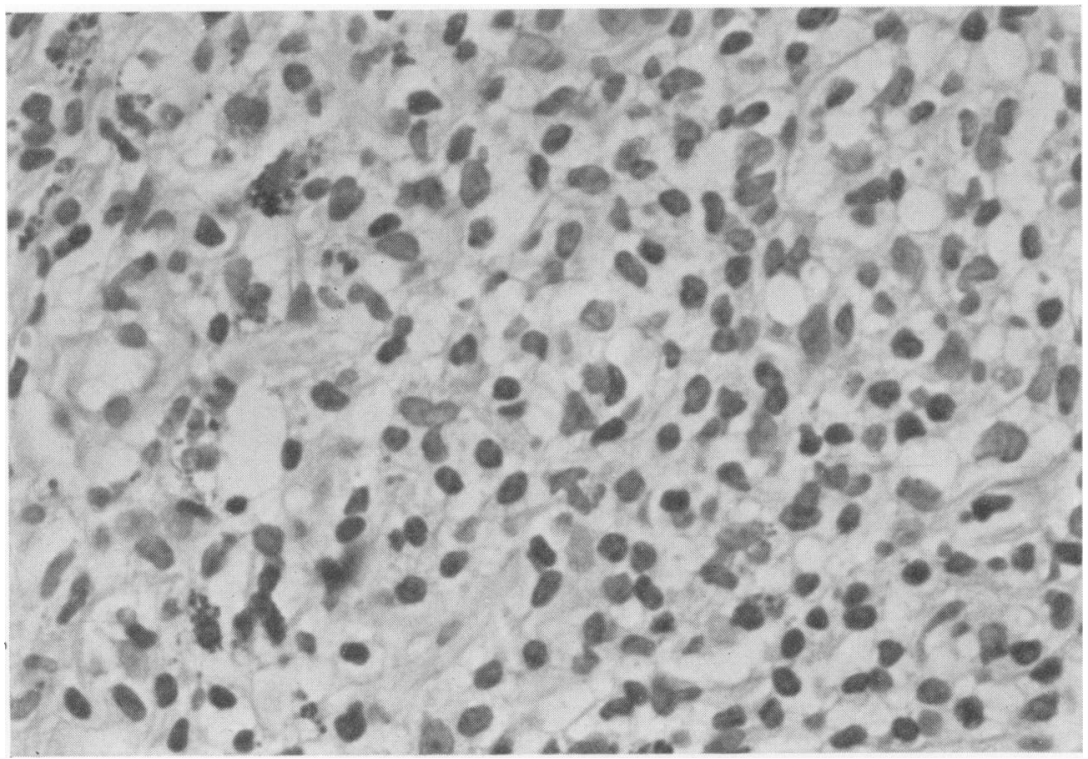

FIG. 4. Skin biopsy $(\times 224)$. Dense pleomorphic cell infiltrate in upper dermis.

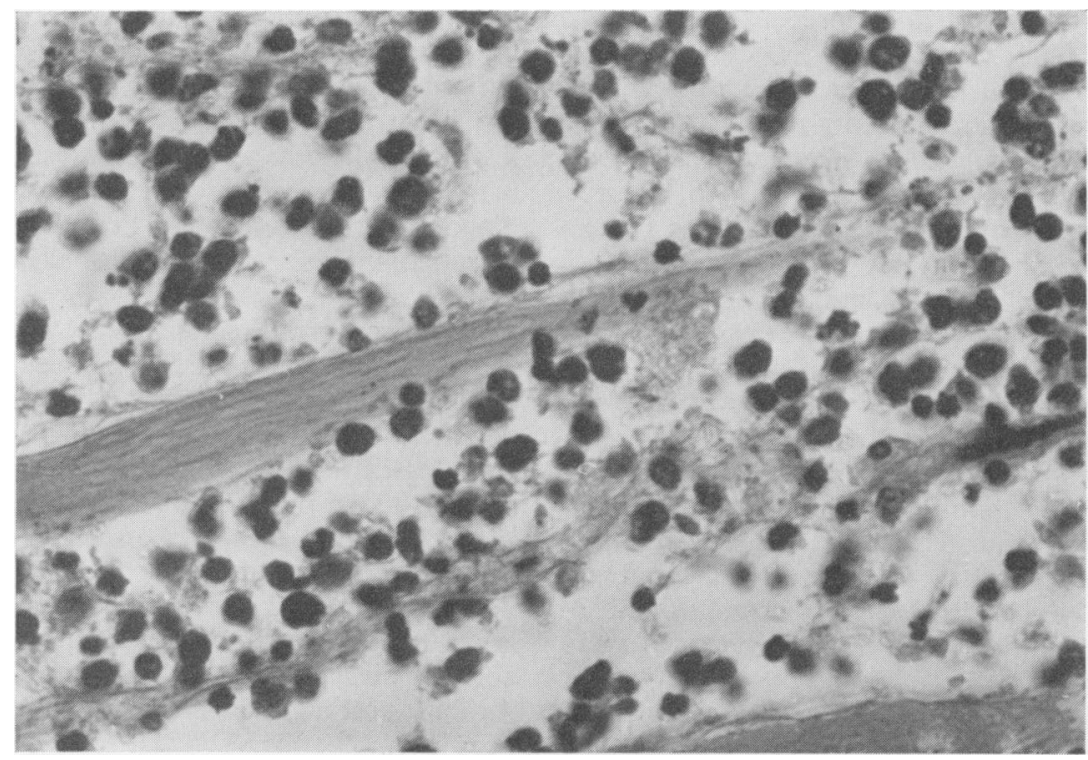

Fig. 5. Psoas muscle $(\times 224)$. Muscle fibres separated by sheets of uniform histiocyte-like cells. 


\section{Discussion}

Much of the confusion over skin reticulosis is due to the terminology employed. Mycosis fungoides can be considered (Samman, 1972) to be a clinical but probably not a distinct pathological entity. It is logical to expect such a reticulosis, at least in a percentage of cases, to become progressively more primitive giving Hodgkin's disease, lymphosarcoma or reticulum cell sarcoma, sometimes with terminal leukaemia. The earlier polymorphous dermal infiltrate becomes simplified (Lever, 1967) as host resistance diminishes with the lymphoma cell becoming the dominant cell type. While some (Milne, 1972) would restrict the term mycosis fungoides to a reticulosis confined clinically and pathologically to the skin, another point of view (Clendenning, 1971) would prefer the term mycosis fungoides to include every malignant reticulosis originating in the skin and to be retained even when spread occurs to other reticulo-endothelial structures such as lymph nodes and bone marrow or to other organs. Such a holistic approach is supported by studies of the outcome of mycosis fungoides. Visceral dissemination was found (Block et al., 1963) in seventeen patients at autopsy. Three other patients with cutaneous changes had no evidence of visceral involvement at post-mortem suggesting that mycosis fungoides is not preceded by visceral lymphoma. It seems likely that mycosis fungoides arises primarily in the skin, not coincidentally with visceral lymphoma. Of 106 cases with biopsy-proved mycosis fungoides (Cyr, Geokas and Worsley, 1966), fourteen showed terminal reticulum cell sarcoma with five having monocytic leukaemia. Fifteen others had lymphosarcoma, six of them also having terminal lymphoblastic leukaemia. Another seven patients had Hodgkin's disease terminally. However, in twenty-three of the forty-two patients from the series who came to autopsy the disease remained as mycosis fungoides throughout its course. The skin lesions in classical mycosis fungoides of the Alibert type are usually non-specific eczematous or psoriasiform plaques of varying colour and bizarre shape which fail to respond to therapy and progress to infiltration and tumours, often with severe pruritus. Diagnostic difficulties centre on the question of what skin lesions indicate a pre-reticulotic state. In the light of experience, a greatly simplified classification has been put forward (Samman, 1972). Conditions which rarely, if ever, progress to reticulosis he would include under the heading of parapsoriasis en plaques (benign type) including such entities as xanthoerythroderma perstans, chronic superficial dermatitis and the small, discrete type of parapsoriasis en plaques. Histology will show only the features of eczema.

The one significant pre-mycotic eruption in his experience is poikiloderma atrophicans vasculare in which he places parapsoriasis lichenoides, atrophic parapsoriasis and the large plaque type of parapsoriasis en plaques. There may be small or large patches of skin involvement as in our patient, often symmetrically involving chest, buttocks and flexures. Definite infiltration indicates the onset of mycosis fungoides or other reticulosis. The upper dermis on histology will show a predominantly lymphocytic infiltrate with excess of reticulum cells and histiocytes-unlike the appearances in a benign lesion.

In conclusion, our patient would appear to have had progressive involvement of head, arms and trunk by poikiloderma atrophicans vasculare over the course of 40 years. Transition to mycosis fungoides probably occurred with the appearance of ulceration. Although fungating tumours were never present, the features were in keeping with those of the tumour stage of mycosis fungoides. With progressive increase in malignancy, systemic spread occurred after 3 years as reticulum cell sarcoma.

\section{Acknowledgments}

We wish to thank Dr R. R. Wilson, Consultant Pathologist, for his help and guidance, Mr G. Illingworth, Consultant Orthopaedic Surgeon, for help in management, and $\mathrm{Mr}$ Fergus -Colman, Department of Medical Illustration, for producing the photographs and photomicrographs.

\section{References}

Block, J.B., Edgcomb, J., Eisen, A. \& VAN ScotT, E.J. (1963) Mycosis fungoides. Natural history and aspects of its relationship to other malignant lymphomas. American Journal of Medicine, 34, 228.

Clendenning, W.E. (1971) Mycosis fungoides. In: Modern Trends in. Dermatology-4 (Ed. by P. Borrie), p. 214 . Butterworth \& Co., London.

Cyr, D.P., Geokas, M.C. \& Worsley, G.H. (1966) Mycosis fungoides. Haematologic findings and terminal course. Archives of Dermatology, 94, 558.

LANE, J.E. (1921) Poikilodermia atrophicans vascularis. Archives of Dermatology and Syphilology, 4, 563.

LANE, J.E. (1923) Poikiloderma atrophicans vasculare. Conclusion of previously reported case. Archives of Dermatology and Syphilology, 8, 373.

LEVER, W.F. (1967) Mycosis fungoides. In: Histopathology of the Skin, 4th edn. p. 757. Pitman Medical Publishing Co., London.

MiLne, J.A. (1972) Lymphoid neoplasms of the skin. In: An Introduction to the Diagnostic Histopathology of the Skin, Edward Arnold, London. 1st edn, p. 230.

RoвB-Smith, A.H.T. (1944) The reticular tissue and the skin. British Journal of Dermatology, 56, 151.

SAmman, P.D. (1964) Survey of reticuloses and pre-mycotic eruptions. A preliminary report. British Journal of Dermatology, 76, 1.

Samman, P.D. (1968) St. John's Hospital reticulosis register. Transactions of the St. John's Hospital Dermatological Society, 54, 200.

Samman, P.D. (1972) Mycosis fungoides. In: Textbook of Dermatology (Ed. by A. Rook, D. S. Wilkinson and F. J. G. Ebling), 2nd edn, p. 1396. Blackwell Scientific Publications, Oxford. 